
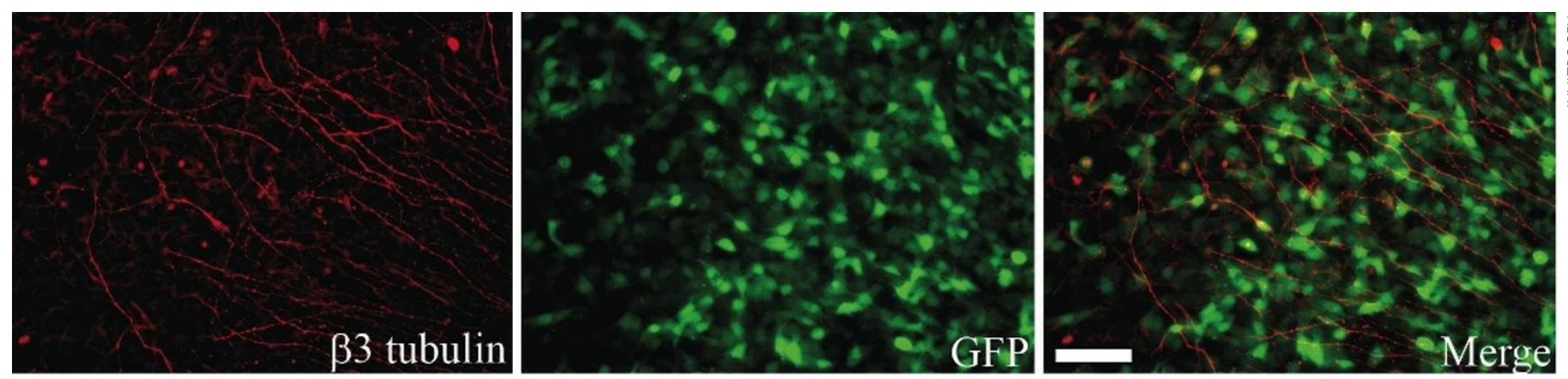

Engineered stem cells differentiated into $\$ 3$ tubulin-expressing neurons (red) while continuing to express green fluorescent protein.

culture. "Cell lines vary in how easy they are to propagate in vitro," says Pera. And Lanza notes that researchers at Advanced Cell Technology can tell embryonic stem-cell lines apart based on the behaviour of the cells in culture.

\section{The mature way to look at stem cells}

Although embryonic stem cells are pluripotent, adult stem cells are multipotent, and can only regenerate specific adult cell types in the body. And for those researchers interested in investigating the potential of adult somatic stem cells, several companies are advancing the idea of providing adult stem cells that have been qualified for culturing on specific media.

Thermo Fisher Scientific supplied media and reagents for culturing adult stem cells, but has now moved into supplying adult stem-cells lines as well. "Our strategy has not only been to create kits to support culturing and differentiation of adult stem cells, but also offer the validated stem cells themselves," says Alain Fairbank, research market manager at Thermo Fisher Scientific. The company now offers four mesenchymal (multipotent stromal cells) stem-cell lines, a haematopoietic (germinal cells from umbilical cord blood) stem-cell line, and a recently discovered multipotent cord-blood cell line that Fairbank says seems to be less restricted in its multi-lineage potential. The advantage of this approach is that all media, reagents and cultureware are certified and validated to work with these specific cell lines. "Our focus has been developing tools that are validated to work together with stem cells," Fairbank says.

Invitrogen of Carlsbad, California, is also "heavily focused on mesenchymal research", says Joydeep Goswami, the company's vicepresident of stem-cell research. "These cells are probably going to be the first non-haematopoietic stem cells to be used for treating patients." In addition to providing adult stem- cell lines, Invitrogen is also actively researching engineered stem cells. "We have been doing a lot of research to convert stem cells themselves into tools," says Goswami. He says that these engineered cell lines act as single-cell reporters providing a visual readout from a live stem cell as it differentiates to separate lineages.

But even as certain adult stem cells become more readily available with well-validated approaches to culturing and differentiation, there is still much work to be done. "Many adult stem-cell populations remain difficult to propagate and expand ex vivo," says Pera. And with the exception of mesenchymal stem cells, he says, this has not changed dramatically in recent years. Although Goswami agrees, he also points to another potential issue. "It is not that adult stem cells have not been isolated, the bigger issue is how do you get well characterized stem cells?" He notes that in some instances researchers will call

\title{
BEYOND THE FLAT WORLD
}

In culture, stem cells rely on signals to differentiate to other cell lineages. Although certain growth factors are known to promote some differentiation programmes, it is now becoming clear that physical interactions between cells and mechanical sensing may also help to promote differentiation. So researchers are developing a variety of three-dimensional (3D) matrices for stem-cell differentiation.

In 2006, Dennis Discher of the University of Pennsylvania in Philadelphia, and his colleagues, demonstrated the potential of the matrix alone to promote differentiation. "The idea was to use synthetic gels with a collagen monolayer to mimic the elasticities of certain tissues and see how the cells respond," says Discher. The work was done under constant serum conditions, without any discriminating soluble factors or growth factors that might promote differentiation in one direction or another. The results showed that by simply varying the elasticity of the matrix, the attached mesenchymal stem cells could undergo either neurogenesis, myogeneis or osteogenesis. They went on to show that once the cells adhere, they begin setting up the stress fibres that actively pull on the adhesions and on the matrix outside. "We showed that the cells feel the matrix and respond to it," says Discher.

Stefan Przyborski is the founder of Reinnervate in Durham, UK, the developer of a new scaffold for routine 3D cell culture. "We create ways of making the in vitro environment a more realistic environment for cell growth," he says. Reinnervate's scaffold is unique, but might seem familiar to biologists who have performed cell culture. "We created a scaffold made of polystyrene, the same material that people currently

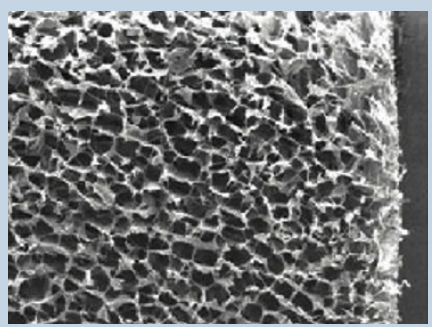

Three-dimensional matrices can promote cell differentiation.

grow their cells on." This is important because researchers know how cells respond to this material in two-dimensional applications. But he also notes that this 3D environment enhances differentiation when compared with cells cultured on twodimensional plates.

Invitrogen of Carlsbad, California has developed Algimatrix, an inert 3D scaffold made of alginate.

"The idea behind Algimatrix is that it provides a framework for cells to reside in that will enable formation of spheroidal structures in a controlled manner," says Mark Powers, a director at Invitrogen. Powers says that when embryonic stem cells form embryoid bodies, they can aggregate into very large structures where the cells on the inside can be oxygen or nutrient limited. But with Algimatrix the aggregates grow to a consistent size and not beyond the size of the pores provided by the scaffold. And since it is an inert scaffold, it actually promotes cell interactions. "A scaffold such as Algimatrix would allow a researcher to culture cells in 3D aggregates to promote differentiation."

It is becoming clear that mechanical interaction has a role in cell differentiation. "What we are really describing is a sense of touch - the cells have no eyes or ears, so they use this sense of touch to tell where they are - this is all part of sensing and responding to the environment," says Discher. N.B. 